\title{
Influence of polarizability on metal oxide properties studied by molecular dynamics simulations
}

\author{
Philipp Beck, ${ }^{1}$ Peter Brommer, ${ }^{2}$ Johannes Roth,${ }^{1}$ and Hans-Rainer Trebin ${ }^{1}$ \\ ${ }^{1}$ Institut für Theoretische und Angewandte Physik (ITAP), Universität Stuttgart, Pfaffenwaldring 57, 70550 Stuttgart, Germany \\ ${ }^{2}$ Département de Physique and Regroupement Québécois sur les Matériaux de Pointe (RQMP), \\ Université de Montréal, C.P. 6128, Succursale Centre-Ville, Montréal, Québec, Canada H3C 3J7
}

(Dated: September 5, 2018)

\begin{abstract}
We have studied the dependence of metal oxide properties in molecular dynamics (MD) simulations on the polarizability of oxygen ions. We present studies of both liquid and crystalline structures of silica $\left(\mathrm{SiO}_{2}\right)$, magnesia $(\mathrm{MgO})$ and alumina $\left(\mathrm{Al}_{2} \mathrm{O}_{3}\right)$. For each of the three oxides, two separately optimized sets of force fields were used: (i) Long-range Coulomb interactions between oxide and metal ions combined with a short-range pair potential. (ii) Extension of force field (i) by adding polarizability to the oxygen ions. We show that while an effective potential of type (i) without polarizable oxygen ions can describe radial distributions and lattice constants reasonably well, potentials of type (ii) are required to obtain correct values for bond angles and the equation of state. The importance of polarizability for metal oxide properties decreases with increasing temperature.
\end{abstract}

\section{INTRODUCTION}

Metal oxides are abundant in many technological applications. Their excellent insulating, thermal isolating and heat resisting properties make them important components in microelectronics and semiconductor engineering. They also play a crucial role in nanotechnology and nanoscience. Modelling these systems in classical atomistic simulations requires effective potentials that describe the interaction between the ions with reasonable accuracy. There exists a wide selection of potential models with various degrees of sophistication, from simple pair potentials to more intricate force fields. All of these have to cope with the long-ranged nature of the Coulomb interaction, which requires special treatment in large-scale simulations. In this work, we will show that certain properties of metal oxides cannot be adequately described in simulation with pure pair potentials.

For crystalline silica $\left(\mathrm{SiO}_{2}\right)$, Herzbach and Binder [1] already showed, that the polarizable force field proposed by Tangney and Scandolo [2] is superior to both the BKS [3] and the fluctuating-charge DCG [4] pair potential models. Recently, polarization effects on different properties of various molten fluorides, chlorides and ionic oxides were described by Salanne and Madden [5]. The authors illustrated the impact of polarizability in several exemplarily selected systems of these material classes and predicted the general importance for structural and dynamic properties.

In this paper we present a systematic comparison of two types of force fields for three different oxides, which only differ by the presence of a polarizable term; the non-electrostatic and Coulomb terms have in each case the same functional form for both force fields. Hence, differences in molecular dynamics (MD) simulation applications can be attributed to polarizability. To obtain a conclusion with a high degree of universality, we present MD simulation studies of both liquid and crystalline structures of silica $\left(\mathrm{SiO}_{2}\right)$, magnesia $(\mathrm{MgO})$ and alumina $\left(\mathrm{Al}_{2} \mathrm{O}_{3}\right)$. Simulations were performed with the MD code IMD $[6,7]$.

For each of the three metal oxides, we used two separately optimized force fields:

(i) Short range interactions of Morse-Stretch (MS) form combined with Coulomb interactions between charged particles.

(ii) Extension of (i) by adding polarizability to the oxygen ions according to the Tangney-Scandolo (TS) potential model [2].

The interactions between charges and/or induced dipoles are long ranged. To handle these electrostatic forces correctly and efficiently, we applied the Wolf summation method [8] in the potential generation as well as in simulations. In several recent studies for metal oxides [9-11], linear scaling of the computational effort in the number of particles could be achieved by using the Wolf summation without significant loss of accuracy.

Details of both TS model and Wolf summation are shown in Sec. II, where also the two force field types are described in detail. In Sec. III, whe present a systematic comparison of the two types of force fields for silica $\left(\mathrm{SiO}_{2}\right)$, magnesia $(\mathrm{MgO})$ and alumina $\left(\mathrm{Al}_{2} \mathrm{O}_{3}\right)$. Discussion and conclusion are given in Sec. IV and Sec. V, respectively.

\section{FORCE FIELDS}

\section{A. Generation}

All force fields were developed with the program potfit $[12,13]$, which generates effective interaction potentials solely from $a b$ initio reference structures. The potential parameters are optimized by matching the resulting forces, energies, and stresses to corresponding firstprinciples values with the force matching method [14]. In 


\begin{tabular}{ccccccc}
\hline \hline$\phi_{\mathrm{S}}^{\mathrm{O}}$ & $D_{\mathrm{Si}-\mathrm{Si}}$ & $D_{\mathrm{Si}-\mathrm{O}}$ & $D_{\mathrm{O}-\mathrm{O}}$ & $\gamma_{\mathrm{Si}-\mathrm{Si}}$ & $\gamma_{\mathrm{Si}-\mathrm{O}}$ & $\gamma_{\mathrm{O}-\mathrm{O}}$ \\
& 0.100032 & 0.100250 & 0.076883 & 11.009449 & 11.670530 & 7.505632 \\
& $\rho_{\mathrm{Si}-\mathrm{Si}}$ & $\rho_{\mathrm{Si}-\mathrm{O}}$ & $\rho_{\mathrm{O}-\mathrm{O}}$ & $q_{\mathrm{Si}}$ & $q_{\mathrm{O}}$ & \\
& 2.375990 & 2.073780 & 3.683866 & 1.799475 & -0.899738 & \\
\hline$\phi_{\mathrm{M}}^{\mathrm{O}}$ & $D_{\mathrm{Mg}-\mathrm{Mg}}$ & $D_{\mathrm{Mg}-\mathrm{O}}$ & $D_{\mathrm{O}-\mathrm{O}}$ & $\gamma_{\mathrm{Mg}-\mathrm{Mg}}$ & $\gamma_{\mathrm{Mg}-\mathrm{O}}$ & $\gamma_{\mathrm{O}-\mathrm{O}}$ \\
& 0.038258 & 0.100261 & 0.065940 & 9.108854 & 10.405694 & 7.962500 \\
& $\rho_{\mathrm{Mg}-\mathrm{Mg}}$ & $\rho_{\mathrm{Mg}-\mathrm{O}}$ & $\rho_{\mathrm{O}-\mathrm{O}}$ & $q_{\mathrm{Mg}}$ & $q_{\mathrm{O}}$ & \\
& 3.384000 & 2.417339 & 3.448060 & 1.100730 & -1.100730 & \\
\hline$\phi_{\mathrm{A}}^{\mathrm{O}}$ & $D_{\mathrm{Al}-\mathrm{Al}}$ & $D_{\mathrm{Al}-\mathrm{O}}$ & $D_{\mathrm{O}-\mathrm{O}}$ & $\gamma_{\mathrm{Al}-\mathrm{Al}}$ & $\gamma_{\mathrm{Al}-\mathrm{O}}$ & $\gamma_{\mathrm{O}-\mathrm{O}}$ \\
& 0.002164 & 1.000003 & 0.000018 & 10.855181 & 7.617923 & 16.719817 \\
& $\rho_{\mathrm{Al}-\mathrm{Al}}$ & $\rho_{\mathrm{Al}-\mathrm{O}}$ & $\rho_{\mathrm{O}-\mathrm{O}}$ & $q_{\mathrm{Al}}$ & $q_{\mathrm{O}}$ & \\
& 5.517666 & 1.880153 & 6.609171 & 1.244690 & -0.829793 & \\
\hline \hline
\end{tabular}

TABLE I. Parameters for the potentials $\phi_{\mathrm{S}}^{0}, \phi_{\mathrm{M}}^{0}$ and $\phi_{\mathrm{A}}^{0}$, given in IMD units set $\mathrm{eV}, \AA$ and amu.

contrast to directly deriving charges and polarizabilities from first principles (as for example done in [15]) or fixing charges to their formal values (as for example done in [16]), these values are also to optimize in potfit in order to optimally reproduce forces, energies and stresses. It was already shown in $[2,10,11]$, that such optimal forcefits yield highly accurate interaction potentials. This also implies that charges and polarizabilities obtained in this way are empirical quantities which need not correspond perfectly to a physical charge or polarizability.

All reference data used in this study were obtained with the plane wave code VASP $[17,18]$. For the optimization, a target function

$$
Z=w_{\mathrm{e}} Z_{\mathrm{e}}+w_{\mathrm{s}} Z_{\mathrm{s}}+Z_{\mathrm{f}}
$$

is minimized. Here, $Z_{\mathrm{e}}, Z_{\mathrm{s}}$ and $Z_{\mathrm{f}}$ are the contributions of the quadratic deviations of energies (e), stresses (s) and forces (f), respectively. $w_{\mathrm{e}}$ and $w_{\mathrm{s}}$ are certain weights to balance the different amount of available data for each quantity. Root mean quare (RMS) errors, $\Delta F_{l}$ (with $l=$ e, s, f), are defined as proportional to the square root of the corresponding $Z_{l}$. Their magnitudes are independent of weighting factors, number, and sizes of reference structures. For the minimization of the potential parameters, a combination of a stochastic simulated annealing algorithm [19] and a conjugate-gradient-like deterministic algorithm [20] is used. Details of the whole optimization approach can be found in Ref. 10 or 12 .

\section{B. Wolf summation}

The electrostatic energies of a condensed system are commonly computed with the Ewald method [21], where the total Coulomb energy of a set of $N$ ions, $U_{\mathrm{qq}}$, is decomposed into two terms $U_{\mathrm{qq}}^{r}$ and $E_{\mathrm{qq}}^{k}$ by inserting a unity of the form $1=\operatorname{erfc}(\kappa r)+\operatorname{erf}(\kappa r)$ with the error function

$$
\operatorname{erf}(\kappa r):=\frac{2}{\sqrt{\pi}} \int_{0}^{\kappa r} d t e^{-t^{2}}
$$

$r$ is the spacial coordinate. The short-ranged erfc term is summed up directly, while the smooth erf term is Fourier transformed and evaluated in reciprocal space. This restricts the technique to periodic systems. However, the main disadvantage is the scaling of the computational effort with the number of particles in the simulation box, which increases as $O\left(N^{3 / 2}\right)$ [22], even for the optimal choice of the splitting parameter $\kappa$.

Wolf et al. [8] designed a method with linear scaling properties $O(N)$ for Coulomb interactions. By taking into account the physical properties of the system, the reciprocal-space term $U_{\mathrm{qq}}^{k}$ is disregarded. In addition, a continuous and smooth cutoff of the remaining screened Coulomb potential $\tilde{U}_{\mathrm{qq}}\left(r_{i j}\right)=q_{i} q_{j} \operatorname{erfc}\left(\kappa r_{i j}\right) r_{i j}^{-1}$ is adopted at $r_{c}$ by shifting the potential so that it goes to zero smoothly in the first two derivatives at $r=r_{c}$. The Wolf method for charges and its extension [9] to dipolar interactions is applied to all force fields the present article deals with. A detailed description of the Wolf summation with focus on its application to dipole contributions can be found in Refs. 9, 10, and 11, where it was successfully applied to silica, magnesia, and alumina.

\section{Type (i): MS + charges}

The potential $\phi_{\mu}^{0}$ (with $\mu=\mathrm{S}, \mathrm{M}, \mathrm{A}$ for silica, magnesia and alumina respectively) consists of a short range part of MS form, and a Coulomb interaction between charged particles. The MS interaction between two atoms $i$ and $j$ at positions $\boldsymbol{r}_{i}$ and $\boldsymbol{r}_{j}$ has the form

$$
\phi_{\mathrm{MS}, i j}=D_{i j}\left[\exp \left[\gamma_{i j}\left(1-\frac{r_{i j}}{\rho_{i j}}\right)\right]-2 \exp \left[\frac{\gamma_{i j}}{2}\left(1-\frac{r_{i j}}{\rho_{i j}}\right)\right]\right],
$$

with $r_{i j}=\left|\boldsymbol{r}_{i j}\right|, \boldsymbol{r}_{i j}=\boldsymbol{r}_{j}-\boldsymbol{r}_{i}$ and the model parameters $D_{i j}, \gamma_{i j}$ and $\rho_{i j}$, which have to be optimized. The Coulomb interaction between two atoms is $U_{\mathrm{qq}, i j}=$ $q_{i} q_{j} r_{i j}^{-1}$. The charge $q_{\nu}$ of each atom type (with $\nu=$ $\mathrm{O}, \mathrm{Si}, \mathrm{Mg}, \mathrm{Al}$ for oxygen, silicon, magnesium and aluminum respectively) is determined during the potential optimization under the constraint of charge neutrality. 
The long-range Coulomb interactions are treated with the Wolf summation method both in force field generation and simulation to obtain $\phi_{\mathrm{qq}, i j}$. The total interaction is obtained by summing over all pairs of atoms:

$$
\phi^{0}=\phi_{\mathrm{MS}}^{0}+\phi_{\mathrm{qq}}^{0}=\sum_{\substack{i, j \\ i>j}}\left(\phi_{\mathrm{MS}, i j}+\phi_{\mathrm{qq}, i j}\right)
$$

With potfit, we generated effective interaction potentials $\phi_{\mu}^{0}$ for silica, magnesia and alumina. Table I shows the corresponding parameters.

\section{Tangney-Scandolo dipole model}

In the TS model [2], the polarizability $\alpha$ of the oxygen atoms is taken into account. The dipole moments depend on the local electric field of the surrounding charges and dipoles. Hence, a self-consistent iterative solution has to be found. In the TS approach, a dipole moment $\boldsymbol{p}_{i}^{n}$ at position $\boldsymbol{r}_{i}$ in iteration step $n$ consists of an induced part due to an electric field $\boldsymbol{E}\left(\boldsymbol{r}_{i}\right)$ and a short-range part $\boldsymbol{p}_{i}^{\mathrm{SR}}$ due to the short-range interactions between charges $q_{i}$ and $q_{j}$. Following Rowley et al. [23], this contribution is given by

$$
\boldsymbol{p}_{i}^{\mathrm{SR}}=\alpha \sum_{j \neq i} \frac{q_{j} \boldsymbol{r}_{i j}}{r_{i j}^{3}} f_{i j}\left(r_{i j}\right)
$$

with

$$
f_{i j}\left(r_{i j}\right)=c_{i j} \sum_{k=0}^{4} \frac{\left(b_{i j} r_{i j}\right)^{k}}{k !} e^{-b_{i j} r_{i j}} .
$$

$f_{i j}\left(r_{i j}\right)$ was introduced ad hoc to account for multipole effects of nearest neighbors and is a function of very short range. $b_{i j}$ is the reciprocal of the length scale over which the short-range interaction comes into play, $c_{i j}$ determines amplitude and sign of this contribution to the induced moment. $\alpha, b_{i j}$, and $c_{i j}$ have to be optimized additionally. Together with the induced part, one obtains

$$
\boldsymbol{p}_{i}^{n}=\alpha_{i} \boldsymbol{E}\left(\boldsymbol{r}_{i} ;\left\{\boldsymbol{p}_{j}^{n-1}\right\}_{j=1, N},\left\{\boldsymbol{r}_{j}\right\}_{j=1, N}\right)+\boldsymbol{p}_{i}^{\mathrm{SR}},
$$

where $\boldsymbol{E}\left(\boldsymbol{r}_{i}\right)$ is the electric field at position $\boldsymbol{r}_{i}$, which is determined by the dipole moments $\boldsymbol{p}_{j}$ in the previous iteration step. Due to its excellent performance and scaling properties (see Fig. 1 and Ref. 24), the TS model was used for more than 50 publications in the past ten years.

\section{E. Type (ii): (i) + dipoles}

Beside charges, electric dipole moments on each oxygen ion are taken into account using the TS model. In addition to the interaction between charges $\left(\phi_{\mathrm{qq}}\right)$, the

\begin{tabular}{llll}
\hline \hline$\mu$ & RMS error & $\phi_{\mu}^{0}$ & $\phi_{\mu}$ \\
\hline $\mathrm{S}$ & $\Delta F_{e}$ & 0.216605 & $0.192220[10]$ \\
& $\Delta F_{s}$ & 0.040983 & $0.034099[10]$ \\
& $\Delta F_{f}$ & 1.866665 & $1.621107[10]$ \\
\hline $\mathrm{M}$ & $\Delta F_{e}$ & 0.075370 & $0.116994[10]$ \\
& $\Delta F_{s}$ & 0.029595 & $0.022774[10]$ \\
& $\Delta F_{f}$ & 0.671468 & $0.529535[10]$ \\
\hline $\mathrm{A}$ & $\Delta F_{e}$ & 0.139316 & $0.049172[11]$ \\
& $\Delta F_{s}$ & 0.055651 & $0.027273[11]$ \\
& $\Delta F_{f}$ & 0.203966 & $0.350653[11]$ \\
\hline \hline
\end{tabular}

TABLE II. RMS errors from the optimization of the force fields $\phi_{\mu}$ and $\phi_{\mu}^{0}$ (with $\mu=\mathrm{S}, \mathrm{M}$, A for silica, magnesia and alumina respectively).

Wolf summation method is also applied [9] to the interaction charge-dipole $\left(\phi_{\mathrm{qp}}\right)$ and dipole-dipole $\left(\phi_{\mathrm{pp}}\right)$. This yields the total interaction

$$
\phi=\phi_{\mathrm{MS}}+\phi_{\mathrm{qq}}+\phi_{\mathrm{qp}}+\phi_{\mathrm{pp}}
$$

In this work, we use the polarizable force fields for silica [10], magnesia [10] and alumina [11] presented in earlier publications. These potentials were also generated with potfit.

It must again be emphasized that while the potentials $\phi_{\mu}^{0}$ and $\phi_{\mu}$ share the same functional form for shortrange and Coulomb interactions, their potential parameters were optimized individually. Otherwise, a comparison would only lead to the trivial result: A potential where some contributions are omitted is no longer accurate.

\section{F. RMS errors and scaling properties}

Although the potentials $\phi_{\mu}$ have three more parameters than the $\phi_{\mu}^{0}(13$ compared to 10$)$, they do not describe the reference data significantly better than the $\phi_{\mu}^{0}$. This is illustrated by the RMS errors, that are shown in Table II. Indeed, there is a trend favoring the polarizable potentials: seven of nine RMS error are better in the case of $\phi_{\mu}$. And in the case of silica and magnesia, the RMS errors are - on average - smaller for $\phi_{\mu}$ than for $\phi_{\mu}^{0}$. For alumina, however, it is the other way round. Thus, the RMS errors are only first indicators of the quality of the generated force field, but they are not able to denote for the practicability of a potential model.

All presented force fields are pure pair term potentials. Hence, simulations are less expensive compared to other approaches with many-body potentials like a threebody interaction approach. Apart from that, considering polarizabiliy takes simulations time, because a new selfconsistent solution for all dipole moments has to be found in each MD time step. Taking dipoles into account slows down simulation by a constant factor (in the case of silica about 2.6). The number of steps in the self-consistency loop is independent of the system size (Fig. 1). Both $\phi_{\mu}^{0}$ 


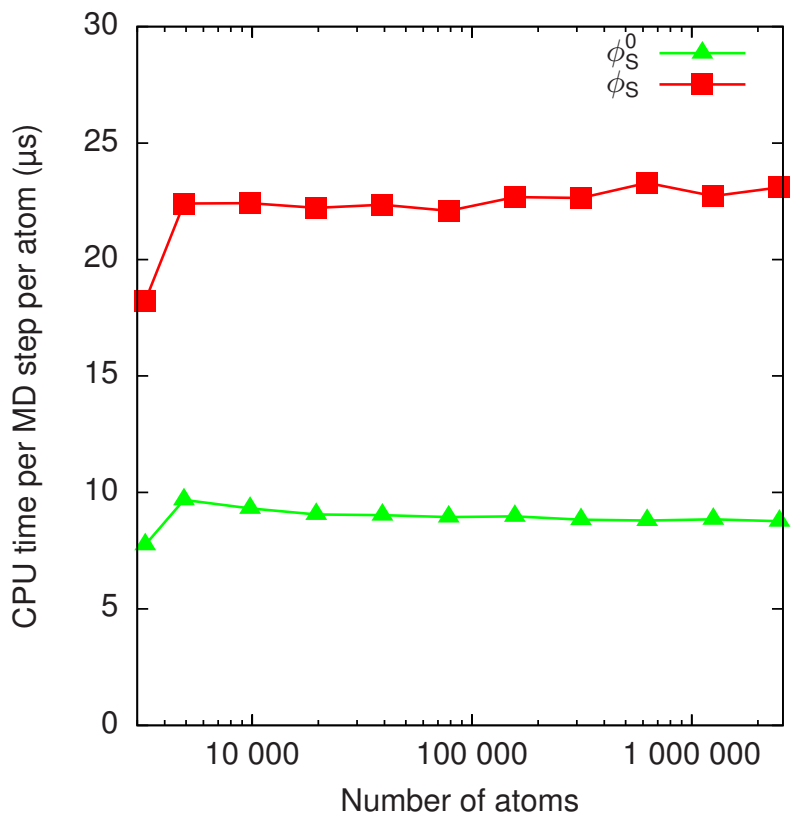

FIG. 1. Scaling of computational effort with system size for $\phi_{S}^{0}$ and $\phi_{S} . \phi_{S}$ also scales linear with system size using Wolf summation, but is slower by a factor of about 2.6 compared to $\phi_{S}^{0}$. The scaling properties of simulations of magnesia and alumina are the same as those of silica: $\phi_{\mu}$ is slower than $\phi_{\mu}^{0}$ by a factor independent of system size.

\begin{tabular}{llll}
\hline \hline & $\mathrm{a}(\AA)$ & $\mathrm{c}(\AA)$ & $\mathrm{E}_{\mathrm{coh}}(\mathrm{eV})$ \\
\hline$\phi_{A}^{\mathrm{O}}$ & 4.87 & 13.24 & 34.71 \\
$\phi_{A}$ & $4.79[11]$ & $12.97[11]$ & $31.85[11]$ \\
Ab initio & $4.78[11]$ & $13.05[11]$ & $32.31[11]$ \\
Experiment & $4.75[26]$ & $12.99[26]$ & $31.8[27]$ \\
\hline \hline
\end{tabular}

TABLE III. Lattice constants and cohesive energy per $\mathrm{Al}_{2} \mathrm{O}_{3}$ unit of $\alpha$-alumina at zero Kelvin obtained with $\phi_{A}$ and $\phi_{A}^{0}$ compared with $a b$ initio results and literature data.

and $\phi_{\mu}$ yield linear scaling with the number of particles due to the Wolf summation. A comparison of the Wolf performance with two mesh-based methods can be found in Ref. 10.

\section{RESULTS}

It is known from Ref. 10 that force fields may also yield qualitative results beyond the range for which they were optimized, however such applications beyond the optimization range should be closely verified. In the following, we focus the tests on the range for the force fields were trained, but we also show results outside this zone to demonstrate the transferability of the potentials.

\begin{tabular}{lllll}
\hline \hline & $a(\AA)$ & $c(\AA)$ & $\mathrm{Si}-\mathrm{O}(\AA)$ & $\mathrm{Si}-\mathrm{O}-\mathrm{Si}$ \\
\hline$\phi_{S}^{0}$ & 4.98 & 5.47 & 1.67 & $139^{\circ}$ \\
$\phi_{S}[10]$ & 5.15 & 5.50 & 1.65 & $148.5^{\circ}$ \\
Theory & $4.97[28]$ & $5.39[28]$ & $1.61[29]$ & $145^{\circ}[29]$ \\
\hline \hline
\end{tabular}

TABLE IV. Lattice constants, $\mathrm{Si}-\mathrm{O}$ bond length and $\mathrm{Si}-\mathrm{O}-\mathrm{Si}$ angle of $\alpha$-quartz at $300 \mathrm{~K}$ compared with theoretical studies.

\section{A. Microstructural properties}

First, the influence of polarizability on microstructural properties is illustrated. The radial distribution functions for liquid silica (4896 atoms) at 3000-6000 K are, in each case, evaluated for 100 snapshots taken out of 100 ps MD runs at the given temperature. The averaged curves are given in Fig. 2. The curves obtained with $\phi_{\mathrm{S}}^{0}$ are similar to the curves of $\phi_{\mathrm{S}}$. The existing slight deviations decrease with increasing temperature, so the polarizability is more important for lower temperatures. For magnesia (see Fig. 3), the radial distribution functions show comparable behaviour: small deviations between $\phi_{M}^{0}$ and $\phi_{M}$, that decrease with temperature. Apparently, the radial distribution in high temperature oxide melts does not require polarizable oxide ions.

A stronger influence of polarizability is observed on bond angles. Fig. 4 (left) depicts the oxygen centered angle distribution at $3000-6000 \mathrm{~K}$ in liquid silica and magnesia, respectively. Both $\phi_{\mathrm{S}}^{0}$ and $\phi_{\mathrm{M}}^{0}$ overestimate the region of lower angles and underestimate the region of higher angles. Potentials with electrostatic dipole moments yields the correct shift of the distributions to slightly higher angles. Again, deviations decrease with increasing temperature. Although $\phi_{\mathrm{A}}$ and $\phi_{\mathrm{A}}^{0}$ were optimized for low-temperature crystalline structures, we probed the behavior for liquid alumina at 3000 K. Fig. 4 (right) shows the oxygen centered angle distribution in alumina compared to a recent $a b$ initio study [25]. Although the trend of shifting angles to higher values by allowing for polarizability is not reproduced in this case, polarizability yields a curve which is in better agreement to $a b$ initio data. These results coincide with Ref. 5, where the authors stated that polarization effects in ionic systems play an important role in determining bond angles.

The electrostatic dipole moments also influence crystalline structure parameters. The lattice constants of $\alpha$ alumina are given in Table III. $\phi_{\mathrm{A}}$ yields an accurate agreement both with $a b$ initio and experimental data, whereas with $\phi_{\mathrm{A}}^{0}$ the lattice constants are overestimated (in each case around $2 \%$ deviation). However, both stabilize the trigonal crystal structure. We also determined lattice constants, $\mathrm{Si}-\mathrm{O}-\mathrm{Si}$ angle and $\mathrm{Si}-\mathrm{O}$ bond length for $\alpha$-quartz at $300 \mathrm{~K}$ (see Table IV), which is outside the optimization range for the $\phi_{\mathrm{S}}$ and $\phi_{\mathrm{S}}^{0}$ potentials. The average relative deviation of all parameters is for both potentials very similar $\left(2.6 \%\right.$ for $\phi_{\mathrm{S}}$ and $2.3 \%$ for $\left.\phi_{\mathrm{S}}^{0}\right)$. On closer inspection, $\phi_{\mathrm{S}}^{0}$ yields more accurate lattice con- 

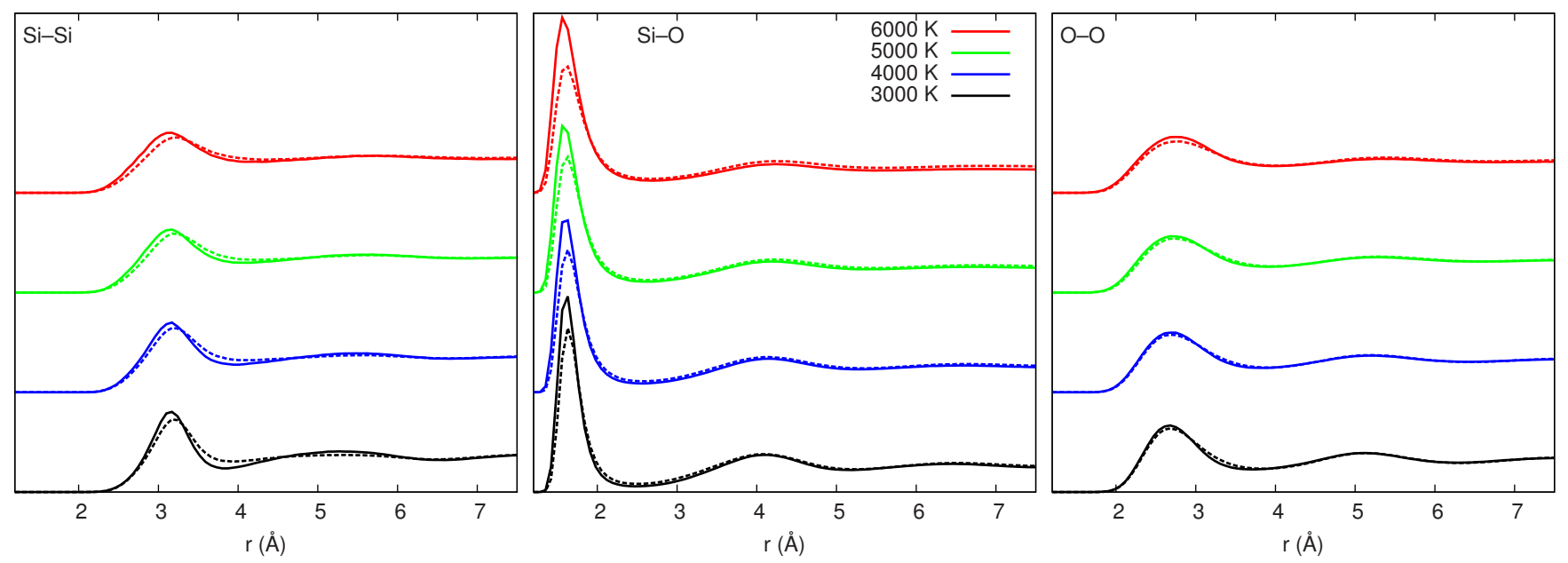

FIG. 2. Normalized radial distribution functions for $\mathrm{Si}-\mathrm{Si}, \mathrm{Si}-\mathrm{O}$ and $\mathrm{O}-\mathrm{O}$ in liquid silica at 3000-6000 K. The solid (dashed) curves belong to $\phi_{\mathrm{S}}\left(\phi_{\mathrm{S}}^{0}\right)$.
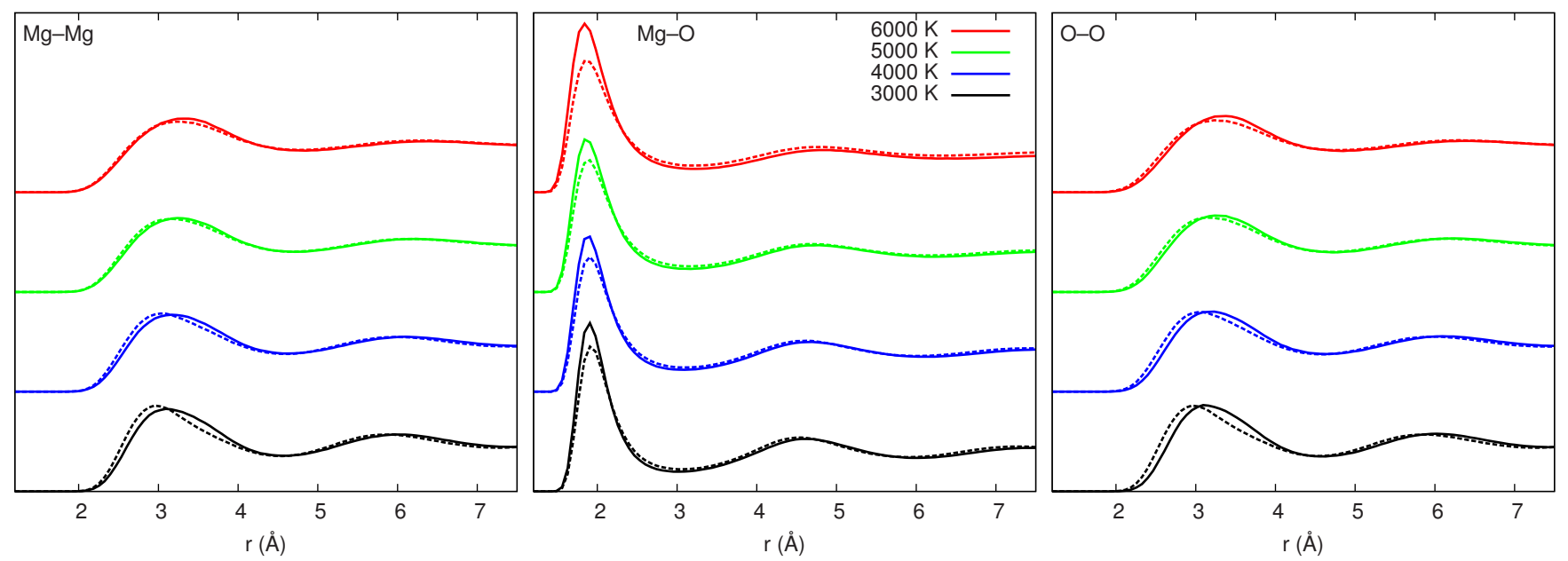

FIG. 3. Normalized radial distribution functions for $\mathrm{Mg}-\mathrm{Mg}, \mathrm{Mg}-\mathrm{O}$ and $\mathrm{O}-\mathrm{O}$ in liquid magnesia at 3000-6000 K. The solid (dashed) curves belong to $\phi_{\mathrm{M}}\left(\phi_{\mathrm{M}}^{0}\right)$.

stants, whereas $\phi_{\mathrm{S}}$ better reproduces the $\mathrm{Si}-\mathrm{O}-\mathrm{Si}$ angle and $\mathrm{Si}-\mathrm{O}$ bond length. This is consistent with Ref. 5 and the results above concerning liquid metal oxides, where also the polarizability is more important for an improved description of bond angles rather than for atomic distances.

\section{B. Thermodynamic properties}

For $\alpha$-alumina, we also calculated the cohesive energy (see Table III). $\phi_{\mathrm{A}}$ coincides with $a b$ initio and experimental results, $\phi_{\mathrm{A}}^{0}$ overestimates the cohesive energy (averaged deviation of $8.3 \%$ ). This clear deviation shows that electrostatic dipole moments have to be taken into account, when probing macroscopical system properties.

To investigate the influence of polarizability on other thermodynamic properties, we show the equation of state of liquid silica (3100 K, see Fig. 5) and magnesia (5000 K and $10000 \mathrm{~K}$, see Fig. 6) respectively. Pressures were obtained as averages along constant-volume MD runs of 10 ps following 10 ps of equilibration. The curves obtained with $\phi_{\mathrm{S}}$ and $\phi_{\mathrm{M}}$ coincide with ab initio results as well as with experiment in the case of silica. The potentials $\phi_{\mathrm{S}}^{0}$ and $\phi_{\mathrm{M}}^{0}$, however, show a clear underestimation of the volume, which illustrates the need for polarizability. The insufficiency of $\phi_{\mathrm{M}}^{0}$ does not decrease with increasing temperature as in the case of microstructural properties. For the equation of state, polarizability has to be taken into account regardless of the simulation temperature.

\section{DISCUSSION}

Apparently, the equation of state of liquid oxides shows the most significant difference between $\phi_{\mu}^{0}$ and $\phi_{\mu}$; the 

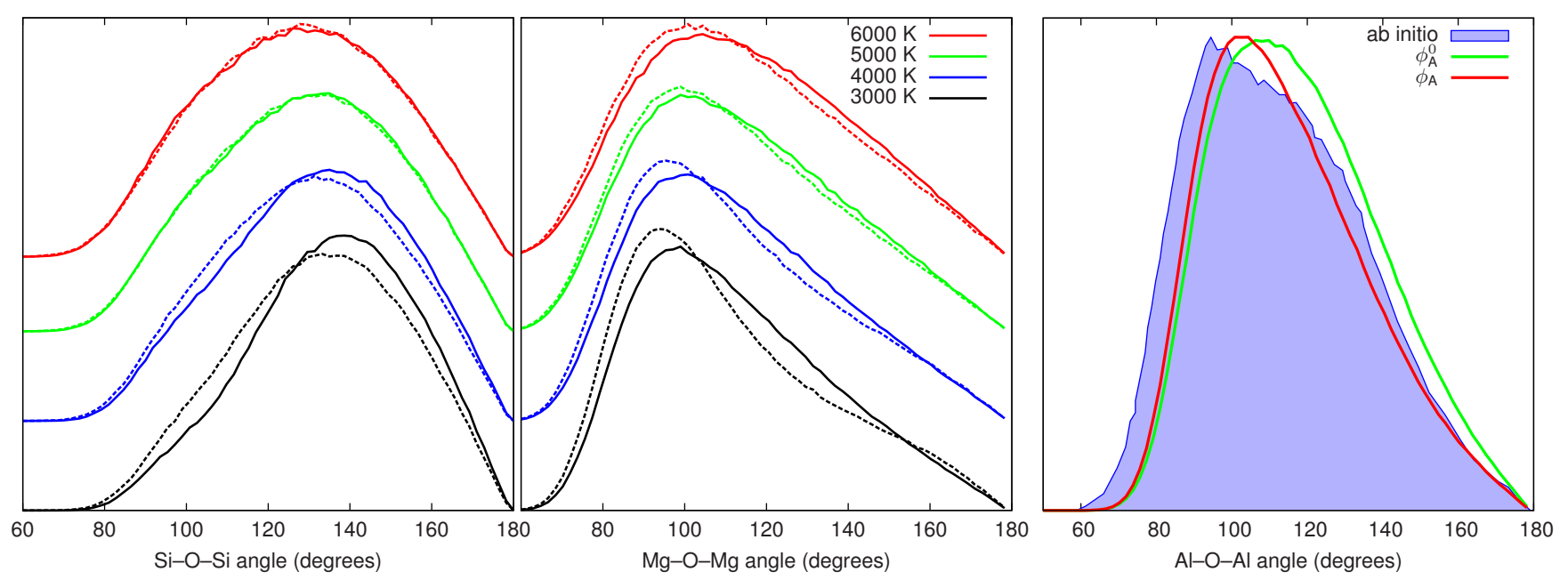

FIG. 4. Left: Normalized oxygen centered angle distributions in liquid silica and magnesia at 3000-6000 K for $\phi_{\mathrm{S}, \mathrm{M}}$ (solid curves) and $\phi_{\mathrm{S}, \mathrm{M}}^{0}$ (dashed curves). $\phi_{\mathrm{S}}^{0}$ yields a shoulder between around 80 and 130 degrees, whereas the band at around 135-170 degrees is underestimated. Similarly, $\phi_{\mathrm{M}}^{0}$ yields a shoulder between around 65 and 95 degrees, whereas the band at around 110-150 degrees is underestimated. Deviations decrease with increasing temperature. Right: The oxygen centered angle distribution in liquid alumina is shown at $3000 \mathrm{~K}$ for $\phi_{\mathrm{A}}$ and $\phi_{\mathrm{A}}^{0}$ and compared to an ab initio study [25].

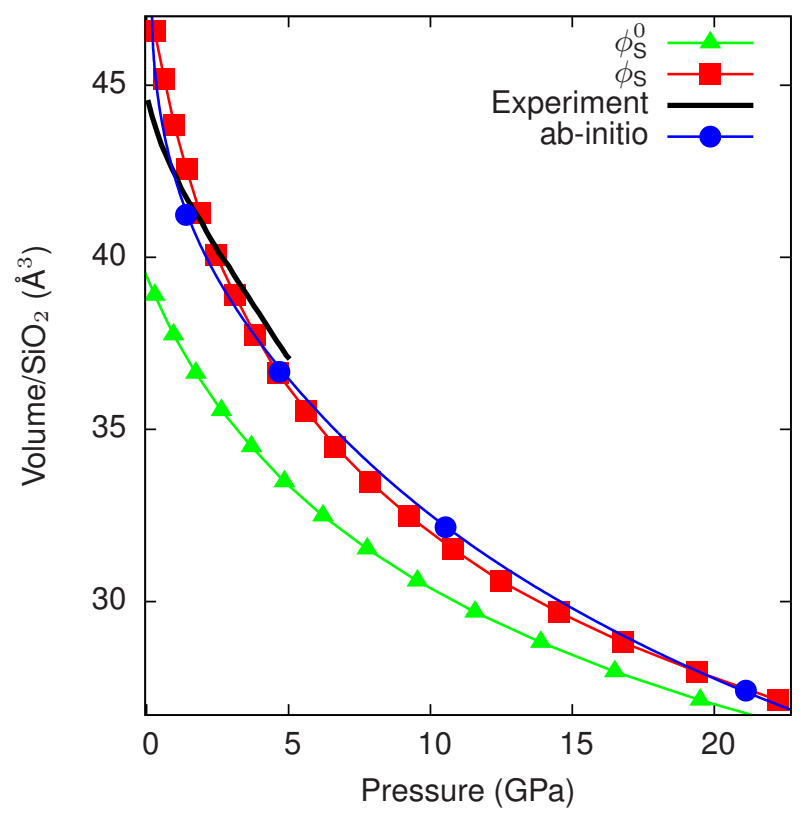

FIG. 5. Equation of state of liquid silica at $3100 \mathrm{~K}$ for $\phi_{\mathrm{S}}[10]$ and $\phi_{\mathrm{S}}^{0}$ compared with experiment [30] and ab initio calculations [31].

potentials without polarizability seem to lack a significant contribution to the pressure. This also applies to the (non-polarizable) BKS force field [3], which underestimates pressure at fixed specific volume by a comparable amount (cf. Ref. 2). The additional pressure in simulations with $\phi_{\mu}$ can however not directly be attributed to dipolar interactions. An analysis of the virial showed that $\phi_{\mathrm{qp}}$ and $\phi_{\mathrm{pp}}$ only contribute about $1.2 \%$ of the total virial (and thus the pressure); the higher pressure for

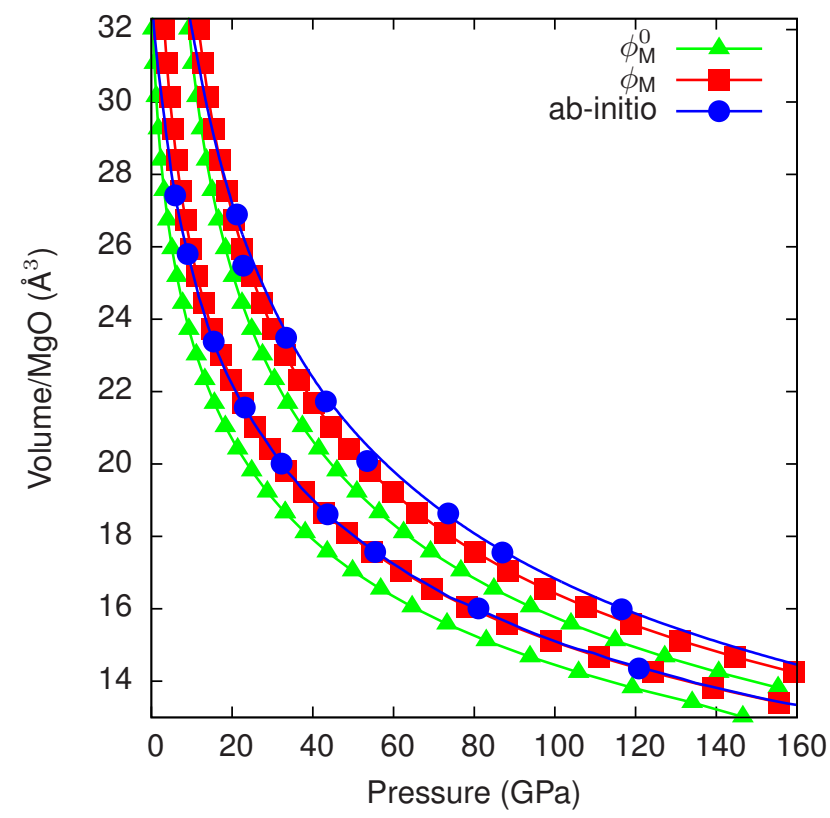

FIG. 6. Equation of state of liquid magnesia at $5000 \mathrm{~K}$ (left three curves) and $10000 \mathrm{~K}$ (right three curves) for $\phi_{\mathrm{M}}$ and $\phi_{\mathrm{M}}^{0}$ compared with ab initio calculations [32].

these systems results almost exclusively from stronger MS and Coulomb contributions to the virial. As the atomic forces are described with comparable precision for both sets of potentials, this implies that the dipolar interaction is required to obtain correct pressures and forces simultaneously, especially as the polarizable force fields have higher absolute values of the atomic charges.

When looking at the parameters of the force fields, it is noticable that in the $\phi_{\mu}^{0}$, the MS potentials are stronger 
at smaller atomic distances: The absolute value of the MS strengh $D_{i j}$ is higher and the stretch length $\rho_{i j}$ (cf. Eq. (3)) is shorter in the non-polarizable potentials. This seems to indicate that in this case, MS is required to describe the atomic interactions for nearest neighbours, while the dipolar interactions provide these contributions for the force fields with polarization.

A better insight is uncovered by inspecting in detail, how accurate the reference $a b$ initio forces and stresses are reproduced by the particular force fields: Fig. 7 depicts scatter plots, where for each single quantity (force component, stress component) the value computed by the potential is plotted against its reference data value. Hence, for perfect matching a point is placed on the bisecting line. As can be seen from the below graphs of Fig. 7 showing the force components of each single atom, both force field types $\phi_{\mu}$ and $\phi_{\mu}^{0}$ yield distributions scattered around the bisecting line. The only difference between $\phi_{\mu}$ and $\phi_{\mu}^{0}$ is how accurate the bisecting line is hit. In the top graphs, however, where the stress components of each configuration are depicted, clear deviations are uncovered: In the silica case, just a slightly worse matching of $\phi_{S}^{0}$ compared to $\phi_{S}$ is visible. But $\phi_{M}^{0}$ produces unnatural meanderings from the optimal matching at the left end of the graph (negative stress component values). The failure becomes even more apparent in the alumina case, where $\phi_{A}^{0}$ underestimates the stresses over the whole data set. To sum up, the scatter plots predict less accuracy for the $\phi_{\mu}^{0}$, when investigating system properties with a strong dependence on stress and pressure.

\section{CONCLUSION}

In summary, we illustrated over a wide range the influence of polarizability on structural and thermodynamic properties in liquid and crystalline systems of silica $\left(\mathrm{SiO}_{2}\right)$, magnesia $(\mathrm{MgO})$ and alumina $\left(\mathrm{Al}_{2} \mathrm{O}_{3}\right)$, by comparing two distinct potentials for each material. As the functional form (but not the parameters) of the shortrange part and the Coulomb term are identical, the de- viations between the results of $\phi_{\mu}$ over $\phi_{\mu}^{0}$ could be associated with the additional dipole terms.

We systematically investigated where the effects of electrostatic dipole moments are more important and how the impact arises from additional interaction mechanisms. The strongest influence of polarizability is observed in macroscopic thermodynamic properties as the equation of state and the cohesive energy. Also on a microscopic scale, the role of dipoles is visible. However, the influences are more relevant for bond angle formation than for atomic distances in both liquid and crystalline structures. The influence always decreases with increasing temperature.

Although the three presented metal oxides differ among each other in their stoichiometric configuration, the influence of polarizability is similar. Hence, it is expected that conclusions can be extended to other metal oxide systems. The results are not estimated to be limited to binary oxides as long as the same interaction mechanisms are dominant. At present, the polarizable force field approach is applied to yttrium doped zirconia [33], where a similar dependency of system properties on polarizability is observed. Statements concerning systems with different interaction mechanisms such as hydrogen bonds in water may exceed the present study.

Previous comparisons of different oxide potentials $[1,5]$ have already shown that polarizable force fields are superior in many aspects. In the present work, we demonstrate directly, for which system properties dipole interactions are important and where the influence is negligible. Especially for the equation of state, simple pair potentials cannot reproduce the pressure-density relationship correctly.

\section{ACKNOWLEDGMENTS}

The authors thank Stephen Hocker for many helpful discussions. Support from the DFG through Collaborative Research Centre 716, Project B.1 is gratefully acknowledged.
[1] D. Herzbach, K. Binder, and M. H. Muser, J. Chem. Phys. 123, 124711 (2005).

[2] P. Tangney and S. Scandolo, J. Chem. Phys. 117, 8898 (2002).

[3] B. W. H. van Beest, G. J. Kramer, and R. A. van Santen, Phys. Rev. Lett. 64, 1955 (1990).

[4] E. Demiralp, T. Cagin, and W. A. Goddard, Phys. Rev. Lett. 82, 1708 (1999).

[5] M. Salanne and P. Madden, Mol. Phys. 109, 2299 (2011).

[6] J. Stadler, R. Mikulla, and H.-R. Trebin, Int. J. Mod. Phys. C 8, 1131 (1997), http://www.itap.physik. uni-stuttgart.de/ imd/.

[7] J. Roth, F. Gähler, and H.-R. Trebin, Int. J. Mod. Phys. C 11, 317 (2000).
[8] D. Wolf, P. Keblinski, S. R. Phillpot, and J. Eggebrecht, J. Chem. Phys. 110, 8254 (1999).

[9] P. Brommer, P. Beck, A. Chatzopoulos, F. Gähler, J. Roth, and H.-R. Trebin, J. Chem. Phys. 132, 194109 (2010).

[10] P. Beck, P. Brommer, J. Roth, and H.-R. Trebin, J. Chem. Phys. 135, 234512 (2011).

[11] S. Hocker, P. Beck, J. Roth, S. Schmauder, and H.-R. Trebin, J. Chem. Phys. 136, 084707 (2012).

[12] P. Brommer and F. Gähler, Modelling Simul. Mater. Sci. Eng. 15, 295 (2007), http://potfit.itap.physik. uni-stuttgart.de/.

[13] P. Brommer and F. Gähler, Phil. Mag. 86, 753 (2006). 

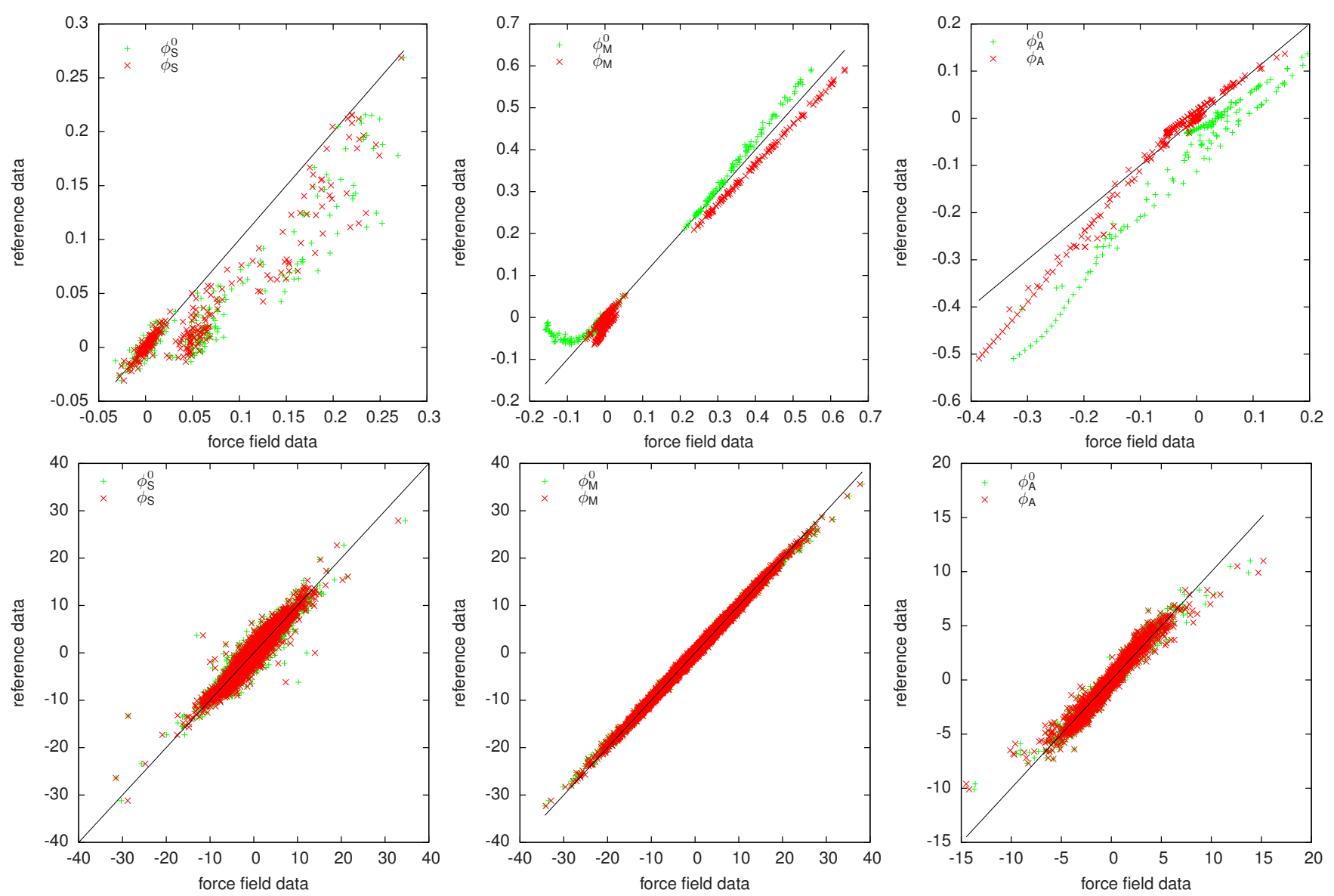

FIG. 7. Values computed by each potential (left silica, central magnesia, right alumina) plotted against its reference data value. A point placed on the bisecting line corresponds to perfect matching. Above: stress components of each configuration (in $\mathrm{MPa}$ ); below: force components of each single atom (in $\mathrm{meV} / \AA$ ).

[14] F. Ercolessi and J. B. Adams, Europhys. Lett. 26, 583 (1994).

[15] R. J. Heaton, and P. A. Madden, J. Chem. Phys. 125, 144104 (2006).

[16] D. Marrocchelli, M. Salanne, P. A. Madden, C. Simon, and P. Turq, Mol. Phys. 107, 443 (2009).

[17] G. Kresse and J. Hafner, Phys. Rev. B 47, 558 (1993).

[18] G. Kresse and J. Furthmüller, Phys. Rev. B 54, 11169 (1996).

[19] A. Corona, M. Marchesi, C. Martini, and S. Ridella, ACM Trans. Math. Softw. 13, 262 (1987).

[20] M. J. D. Powell, Comp. J. 7(4), 303 (1965).

[21] P. P. Ewald, Ann. Phys. (Leipzig) 64, 253 (1921).

[22] D. Fincham, Mol. Sim. 13, 1 (1994).

[23] A. J. Rowley, P. Jemmer, M. Wilson, and P. A. Madden, J. Chem. Phys. 108, 10209 (1998).

[24] J. R. Kermode, S. Cereda, P. Tangney, and A. De Vita, J. Chem. Phys. 133, 094102 (2010).

[25] A. K. Verma, P. Modak, and B. B. Karki, Phys. Rev. B 84, 174116 (2011).
[26] P. Villars and L. D. Calvert, Pearson's Handbook of Crystallographic Data for Intermetallic Phases, 2nd Edition, Vol. I (ASM International, Materials Park, Ohio, 1991) p. 970.

[27] R. C. Weast, ed., CRC Handbook of Chemistry and Physics (CRC Press, Boca Raton, FL, 1983).

[28] G. V. Gibbs, A. F. Wallace, D. F. Cox, R. T. Downs, N. L. Ross, and K. M. Rosso, ammin 94, 1085 (2009).

[29] G. V. Gibbs, D. Jayatilaka, M. A. Spackman, D. F. Cox, and K. M. Rosso, jpca 110, 12678 (2006).

[30] G. A. Gaetani, P. D. Asimow, and E. M. Stolper, Geochim. Cosmochim. Acta 62, 2499 (1998).

[31] B. B. Karki, D. Bhattarai, and L. Stixrude, Phys. Rev. B 76, 104205 (2007).

[32] B. B. Karki, D. Bhattarai, and L. Stixrude, Phys. Rev. B 73, 174208 (2006).

[33] A. Irmler, P. Beck, J. Roth, and H.-R. Trebin, Ab initio based polarizable $O(N)$ force field for yttrium doped zirconia (unpublished). 